\title{
Die Schweiz und das UNO-System : die 21. FAO-
} Tagung

La Suisse et le système onusien : la $21^{\text {ème }}$ Conférence de la FAO

Daniel Fino

\section{(2) OpenEdition}

1 Journals

\section{Electronic version}

URL: http://journals.openedition.org/sjep/956

DOI: 10.4000/sjep.956

ISSN: 1663-9677

\section{Publisher}

Institut de hautes études internationales et du développement

\section{Printed version}

Date of publication: 31 août 1982

Number of pages: $267-273$

ISSN: $1660-5926$

\section{Electronic reference}

Daniel Fino, «Die Schweiz und das UNO-System : die 21. FAO-Tagung", Schweizerisches Jahrbuch für Entwicklungspolitik [Online], 2 | 1982, Online erschienen am: 24 Januar 2013, abgerufen am 08 September 2020. URL : http://journals.openedition.org/sjep/956 ; DOI : https://doi.org/10.4000/sjep. 956

(c) The Graduate Institute 


\title{
DIE SCHWEIZ UND DAS UNO-SYSTEM : DIE 21. FAO-TAGUNG
}

\author{
Daniel Fino
}

\section{LA SUISSE ET LE SYSTEME ONUSIEN : LA 21ème CONFERENCE DE LA FAO}

Résumé : La Suisse, bien que n'étant pas membre de l'ONU, fait partie de la plupart des organisations et conférences groupées dans le système des Nations Unies, et, entre autres, de la FAO. L'auteur aborde ici les problèmes institutionnels qui ont surgi lors de la dernière conférence de la $\mathrm{FAO}$, particulièrement à propos de l'efficacité de cette organisation, du montant du budget, et des modalités de contrôle des projets et autres activités menées par la FAO. La Suisse, en compagnie de cinq autres pays industriels, a voté contre le budget présenté par le directeur. Elle a justifié cet acte par le souci de rendre à la FAO une plus grande efficacité, et de lutter contre les coûts administratifs, jugés beaucoup trop hauts. La plupart des pays en voie de développement ne suivaient pas cette analyse et ont donc adopté le budget, à une confortable majorité. Les pays industriels ont également tenté de faire admettre le principe d'évaluations externes à I'organisation. La direction de la FAO et la plupart des pays en voie de développement y sont plutôt opposés, jugeant le contrôle interne suffisant. La discussion a également porté sur le Technical Cooperation Programme, dont les pays industriels jugent qu'il a dépassé ses buts premiers en ne se bornant pas à traiter les cas d'urgence, et en ne se préoccupant pas assez des pays les plus pauvres. L'auteur conclut en approuvant I'attitude de "participation critique" dont fait preuve la Suisse à l'égard de la FAO; il y voit un moyen pour ce pays de participer activement au processus de formation de l'opinion, tant à la FAO qu'au sein d'autres organisations internationales.

In Kürze stehen wir mitten in der öffentlichen Debatte um den Beitritt der Schweiz zur UNO. Als wichtiger, oft vergessener Aspekt in dieser Diskussion muss dabei zunächst angeführt werden, dass die Schweiz bereits Vollmitglied der meisten Unter- und Sonderorganisationen der UNO ist.

Worin besteht der Beitrag der Schweiz in diesen Organisationen? Was ist ihre Haltung zu den verschiedenen Problemen? Kann ein Kleinstaat wie die Schweiz unter den über 150 Nationen überhaupt eine Rolle spielen und einen Einfluss ausüben? Diese und ähnliche Fragen werden sich auch in der Debatte 
um den UNO-Beitritt stellen.

Ohne den Anspruch erheben zu wollen, diese Fragen umfassend beantworten zu können, werfen wir im Folgenden einen kurzen Blick auf eine der Spezialorganisationen der UNO, die Organisation für Ernährung und Landwirtschaft, besser bekannt unter der Abkürzung FAO.

Unsere Wahl fiel aus verschiedenen Gründen auf die FAO. Zunächst ist die Schweiz praktisch sei deren Gründung im Jahre 1945 Mitglied (d.h. seit 1947). Dann handelt es sich bei der FAO um die grösste Organisation, die dem Hunger in der Welt den Kampf angesagt hat. Schliesslich wurden während der 21. FAO-Tagung (7. bis 21. November 1981 in Rom) wichtige Probleme aufgeworfen, die gerade jetzt in der Debatte um den schweizerischen UNOBeitritt immer wieder zur Sprache kommen (Budgethöhe, Verwaltungskosten, Leistungsfähigkeit der Organisation, usw.) und zu denen die Schweizer Delegation (1) engagiert Stellung bezogen hat.

In der gesamten zweiwöchigen Debatte nahmen die eher praktischen Fragen einen zentralen Platz ein. Wir werden uns im Folgenden auf einige der organisatorischen und institutionellen Probleme beschränken, da in der breiten Schweizer Oeffentlichkeit zu diesen Fragen eher Kontroversen bestehen als zur allgemeinen Problematik der Welternährungslage und der Aktualität des Hungerproblems.

Zunächst ein paar allgemeine Angaben zur FAO. Die FAO ist eine autonome Organisation des UNO-Systems mit Sitz in Rom. Hauptziele der Organisation sind die Hungerbekämpfung in der Welt sowie die Verbesserung der Ernährungssituation und der Lebensqualität im allgemeinen, was mittels einer gesteigerten Agrarproduktion und einer besseren Verteilung der landwirtschaftlichen Erzeugnisse verwirklicht werden soll. Die FAO macht die Weltöffentlichkeit auf die Probleme des Hungers und der Ernährungssicherheit aufmerksam, sammelt und verbreitet Informationen zu Fragen der Nahrungsmittelproduktion, der Landwirtschaft, des Forstwesens und der Fischerei, berät die Regierungen bei der Lösung ihrer Produktionsprobleme, verwaltet gemeinsam mit dem UNO-Generalsekretariat das Welternährungsprogramm (Nahrungsmittelhilfe), schaltet sich in Notstandssituationen mit Projekthilfe ein, verwirklicht Entwicklungsprojekte im Rahmen der technischen Zusammenarbeit und vieles andere mehr.

Die alle zwei Jahre stattfindende Konferenz verabschiedet das Arbeitsprogramm und das Budget und nimmt Wahlen vor. Jedes Land verfügt über eine Stimme, unabhängig von seiner Grösse und seinen Beitragszahlungen. Die Konferenz wählt den FAO-Rat, der dem ständigen Sekretariat vorsteht (ca. 4.000 Angestellte). Das reguläre Budget für 1980-81 betrug 279 Mio USDollar. Darüberhinaus verwaltet die FAO zwischen 500 und 600 Mio USDollar an Fremdgeldern, die ihr von der UNO und Mitgliedsländern direkt anvertraut werden.

Der feste Beitrag der Schweiz am regulären Budget umfasst 1,28\%; bei den 
Spezialaktionsprogrammen (extrabudgetäre Mittel) steht die Schweiz mit ihren Beiträgen an sechster Stelle.

Einige der strittigen Punkte in der und um die FAO sind unter anderem die Rolle der FAO innerhalb des UNO-Systems sowie die Koordinierung der von den verschiedenen Organisationen auf dem Gebiet der Landwirtschaft und der Ernährung durchgeführten Aktionen, die interne Verwaltung der FAO und, damit verbunden, die Leistungsfähigkeit der Organisation, die Höhe des Budgets, die Ueberprüfungsmodalitäten der FAO-Unternehmungen und der Umfang der Nahrungsmittelhilfe (2).

Die Verabschiedung des Budgets an der letzten Konferenz gab zu einigen Diskussionen Anlass. Von der FAO wurde für 1982-83 ein Budget von 367 Mio US-Dollar vorgeschlagen (1980-81 : 279 Mio US-Dollar).

In der Schlussabstimmung stimmten fünf Industrieländer, darunter die Schweiz, gegen die Verabschiedung des Budgets, neun Länder enthielten sich der Stimme und 110 Länder nahmen das Budget an. Die fünf NeinStimmer gehören zwar zu den grössten Beitragszahlern (über $50 \%$ ), konnten wegen des herrschenden Prinzips "one land, one vote" die Verabschiedung des Budgets jedoch nicht verhindern.

In einer gemeinsamen Erklärung führten neun Indistrieländer, darunter die Schweiz, aus, dass ihre Haltung nicht als eine Verringerung ihrer Anstrengungen im Kampf gegen den Hunger zu verstehen sei. In der Erklärung heisst es weiter: “Wir wollen vermehrt die Eigenanstrengungen der Entwicklungsländer zur Erhöhung ihrer Nahrungsmittelproduktion unterstützen (...). Die verschiedenen Programme der Vereinten Nationen, die sich mit Ernährung und Landwirtschaft befassen, müssen überarbeitet werden, um Doppelspurigkeit zu vermeiden und um die Zweckmässigkeit und Leistungsfähigkeit dieser Programme zu erhöhen (...). Der Kampf gegen den Hunger lässt sich nicht durch höhere Verwaltungskosten gewinnen" (3). Die Unterzeichner dieser Erklärung schlugen vor, die FAO-Strukturen zu reorganisieren, neue Prioritäten festzulegen sowie leistungsunfähige Programme zu ersetzen.

Das Eidg. Volkswirtschaftsdepartement hat die Haltung der Schweizer Delegation an der FAO-Tagung durch eine Pressemitteilung bestätigt und begründet. Unter den gegenwärtigen wirtschaftlichen Umständen, heisst es im Communiqué, könne dem vorgeschlagenen Budget nicht zugestimmt werden. Das Nein zum Budget sei jedoch nicht mit einem Nein zur FAO und deren Anstrengungen, welche die Schweiz und weiterhin unterstützen werde, gleichzusetzen. Ein Vertreter der Schweizer Delegation erklärte ausserdem, dass die Verwaltungskosten im Rahmen der Entwicklungszusammenarbeit allgemein zu hoch seien. Rechnet man beispielsweise bei FAO-Projekten, die für die Vereinten Nationen durchgeführt werden, den FAO-Ausgaben diejenigen des UNDP (4) und des Finanzierungslandes hinzu, so komme man ohne weiteres auf $30 \%$ und mehr des Projektbudgets.

Die Haltung der grossen Beitragszahler und der Schweiz war demnach ein- 
deutig auf mehr Sparen, einen besseren Einsatz der Mittel sowie eine erhöhte Leistungsfähigkeit ausgerichtet. Sowohl in Bezug auf die allfällige Budgeterhöhung als auch im Hinblick auf eine Verbesserung der Leistungsfähigkeit wurde eine vermehrte Ueberprüfung der FAO-Tätigkeiten, wie zum Beispiel der Dezentralisierungsprogramme und der Programme der technischen Zusammenarbeit, verlangt.

Wie die Budgetschlussabstimmung gezeigt hat, waren die meisten Entwicklungsländer mit der Haltung der Nein-Stimmer nicht einverstanden. Sie wurden dabei vom Generaldirektor der FAO, E. Saouma, unterstützt, welcher in der Debatte erklärte, er stützte sich bei der vorgeschlagenen bescheidenen Budgeterhöhung auf die verschiedenen Erklärungen anlässlich der letzten grossen Konferenzen (Ottawa, Melbourne, Cancun usw.), wonach der Erhöhung der landwirtschaftlichen Produktion der absolute Vorrang gegeben werden müsste. Die FAO könnte ihre Aufgabe in diesem Sinne nur erfüllen, wenn ihr auch die entsprechenden Mittel zur Verfügung stünden.

Fiel die Abstimmung über die Verabschiedung des Budgets zugunsten des Generaldirektors aus, so unterlag dieser in der Abstimmung über die Beibehaltung/Senkung des Ansatzes für die Deckung der Verwaltungskosten von Treuhandprojekten. Der Generaldirektor war für die Beibehaltung des Ansatzes von 14\%. Die Versammlung beschloss jedoch die Senkung auf 13\%, so wie dies auch für das UNDP vorgesehen ist. Ein Vertreter der Schweizer Delegation hielt in einer Intervention fest, dass es für die Schweiz, unter anderem wegen der dieser Frage gegenüber kritisch eingestellten öffentlichen Meinung, praktisch unmöglich wäre, einen höheren Ansatz als $13 \%$ zu akzeptieren.

Was die finanziellen Fragen im allgemeinen anbelangt, bezog die Schweiz sehr engagiert Stellung und trat, wie die meisten anderen Industrieländer, für eine rationellere Verwendung der FAO-Gelder ein. Einerseits ist es in der Tat einleuchtend, dass in einer Zeit der allgemeinen Mittelverknappung (starke Verschuldungsquoten, Haushaltsdefizite usw.) auch innerhalb der internationalen Organisationen der Einsatz der Mittel vermehrt überwacht und der Verschwendung Einhalt geboten wird. Andererseits ist die Haltung der Entwicklungsländer, für die die aufgebrachten Mittel nicht ausreichen, durchaus zu rechtfertigen und zu verteidigen. Deren Forderung nach mehr Unterstützung muss mit erhöhten Eigenanstrengungen und “internen" Massnahmen in diesen Ländern selbst einhergehen. Ein wichtiger Aspekt dabei ist des "Produzentenpreis" für landwirtschaftliche Erzeugnisse. Das Problem der "Produzentenpreises" wurde von der FAO in den letzten Jahren vielleicht zu wenig angegangen, vor allem aus "taktisch-diplomatischen" Gründen. Die FAO hat sich hingegen immer wieder für die internationalen Abkommen stark gemacht, welche auch an der 21. Tagung erneut zur Sprache kamen.

In engem Zusammenhang mit den finanziellen Aspekten steht, wie schon angedeutet, der Ruf nach einer verstärkten Ueberprüfung der FAO-Tätigkeit. Zur Debatte stand dabei nicht nur, welche Themen genauer überprüft werden 
sollten, sondern auch die Art une Weise einer solchen Ueberprüfung. Die Industrieländer sind der Ansicht, dass die FAO-Unternehmungen vermehrt extern zu überprüfen wären und dass somit die internen Evaluierungen nicht ausreichten. Anders gesagt, die Industrieländer vertrauen den FAO-eigenen Kontrollmechanismen nicht, und ein gewisser Vertrauensschwund kann in der Tat nicht geleugnet werden.

Die FAO-Direktion sowie die meisten Entwicklungsländer sind externen Ueberprüfungen eher abgeneigt. Sie argumentieren dabei, dass die Evaluierungsverantwortung den Empfängerländern zukomme und die schon bestehenden Kontroll- und Ueberprüfungsmechanismen genügten.

Die Schweizer Delegation gesteht der FAO zu, über ein zweckmässiges internes Instrument zu verfügen. Sie meint jedoch, dass das bestehende System auch Mängel aufweise, was die FAO übrigens anerkennte. Zusätzliche Massnahmen, wie externe Ueberprüfungen in bestimmten Fällen mit weitreichenden Konsequenzen, könnten die bestehenden Evaluierungsmechanismen wertvoll ergänzen.

Ein Thema, welches für eine Ueberprüfung in Frage kommt, ist beispielsweise das Dezentralisierungsprogramm der FAO. Die FAO möchte die Zahl ihrer Ländervertretungen von 62 auf 74 erhöhen. Dabei steht nicht das Prinzip der Dezentralisierung an sich zur Diskussion, sondern man ist sich über den Zeitpunkt einer solchen Ausdehnung nicht einig. Die Entwicklungsländer möchten eine sofortige Einführung des Programms, während die Industrieländer Wert darauf legen, vorerst die Tätigkeit der bestehenden Büros zu überprüfen und darüberhinaus jene mit den jeweiligen UNDP-Vertretern in den einzelnen Ländern besser in Uebereinklang zu bringen.

Die Schweizer Delegation ist dem Dezentralisierungsprogramm gegenüber positiv eingestellt, jedoch unter der Bedingung, dass die Ländervertreter mit operationellen und administrativen Kompetenzen ausgestattet werden. Während der Konferenz warf die Schweiz verschiedene Fragen auf, zu denen die FAO-Verantwortlichen zum Teil ausführlich Stellung bezogen (Liste der zusätzlich geplanten Büros, Aufgabenbereich und Kompetenzen der FAOLändervertreter usw.).

In Bezug auf die Zusammenarbeit mit den UNDP-Vertretern in den einzelnen Ländern vertritt die FAO den Standpunkt, dass das Empfängerland in erster Linie für die Koordinierung der externen Hilfe verantwortlich sei. Für das UNDP hingegen müssten, gemäss Beschluss der UNO-Vollversammlung, die multilateralen Aktionen unter seinem Patronat aufeinander abgestimmt sein (5).

Was die Ueberprüfung der Projekte im Feld betrifft, so ist einer der strittigen Punkte beispielsweise die Rolle und der Umfang des "Technical Cooperation Programme" (TCP). Das TCP wurde 1976 gegründet, um der FAO die Möglichkeit zu geben, in Notstandssituationen schnell einzugreifen. Dabei bestand nicht die Absicht, den Charakter der FAO, die ja eine ausführende 
Organisation ("executing agency") ist, zu verändern. Bis 1981 wurden ungefähr 1.200 Projekte verwirklicht und für 1982-83 sind dafür 47 Mio USDollar (d.h. 12,85\% des regulären Budgets) vorgesehen. Die Entwicklungsländer schätzen das TCP sehr, weil sich die oft langwierigen Verfahren bei Projektverwirklichungen im Rahmen des TCP verkürzen. Die Industrieländer vertreten hingegen die Ansicht, das TCP habe seine ursprüngliche Zielsetzung (d.h. schnelles Eingreifen in Notstandssituationen) verfehlt und berücksichtigte zu wenig die ärmsten Länder. Nach Meinung der Schweizer Delegation sollte das TCP beibehalten werden, da es eine wichtige Funktion erfülle; allerdings müssten Verbesserungen eingeführt werden. So sollten zum Beispiel die Auswahlkriterien transparenter sein; eine genaue Liste der bewilligten Projekte wäre zu führen; und die Ausdehnung des TCP wäre vorerst aufgrund der bereits in der Budgetdebatte angeführten Argumente zurückzustellen.

Mit diesen kurzen Ausführungen wollten wir einen kleinen Einblick in das "Innenleben" der FAO geben und die Rolle der Schweiz an der 21. FAOTagung hervorheben. Auch wenn wir die gesamte Debatte wiedergegeben hätten - es handelte sich in der Tat um hunderte von Interbentionen -, steht man bei der Beurteilung einer solchen Organisation unweigerlich vor einem Dilemma. Einerseits fragt man sich, ob alle diese langwierigen und aufwendigen Debatten überhaupt eine Wirkung erzielen und inwiefern sich ein solcher Apparat wie der FAO, mit all seinen Widersprüchen und politischen Zwängen, der Lösung des Hungerproblems überhaupt einen Schritt nähern kann. Andererseits stellt sich die Frage, ob es zum bestehenden System internationaler Absprachen, sei es auf der politischen (z.B. Friedenssicherung der UNO) oder der wirtschaftlichen Ebene (z.B. Agrarpreisabkommen) überhaupt eine Alternative gibt. Auch wenn das bestehende System Mängel aufweist, so ist ein anderer Weg grundsätzlich kaum vorstellbar.

In diesem Dilemma scheint uns der Weg der "kritischen Partizipation", den die Schweiz innerhalb der FAO eingeschlagen hat, gangbar und durchaus sinnvoll zu sein. Es ist unmöglich, aufgrund der angeführten Beispiele oder sämtlicher Interventionen der Schweizer Delegation den Einfluss der Schweiz an der FAO-Tagung zu messen. Aber es wird deutlich, dass sich die Schweiz an der Kontroverse um die zukünftige Ausrichtung der $\mathrm{FAO}$ aktiv beteiligt und zu verschiedenen Fragen eingeschaltet hat. Durch diese Beteiligung am Meinungsbildungsprozess kann die Schweiz, sowohl in der FAO wie in anderen internationalen Organisationen, eine wichtige Rolle spielen.

\section{ANMERKUNGEN}

1. Die Schweizer Delegation an der 21. FAO-Tagung, geleitet von J.C. Piot, Direktor des Bundesamtes für Landwirtschaft (BAL), bestand aus sechs Personen : drei Vertretern des BAL, einem Vertreter der Direktion für Entwicklungszusammenarbeit und humani- 
täre Hilfe, einem Vertreter des Eidg. Departements für auswärtige Angelegenheiten und einem Vertreter der Schweizer Botschaft in Rom.

2. Noch wenige Monate vor der Konferenz verlangte der Generaldirektor eine Erhöhung der Nahrungsmittelhilfe von ca. 9 Mio Tonnen (1981) auf 18 Mio Tonnen (1985) und sogar auf 30 Mio Tonnen für 1990. Ohne die Nahrungsmittelhilfe in wirklichen Notstandssituationen in Frage zu stellen, ist man sich heute doch mehr und mehr der negativen Auswirkungen dieser Hilfe auf die Preisgestaltung auf den lokalen Märkten, die Ernährungsgewohnheiten, die dringend erforderlichen Agrarreformen usw. bewusst. Uebrigens hat der Welternährungsrat in diesem Zusammenhang verschiedene Varianten ausgearbeitet, um die Nahrungsmittelhilfe vermehrt in die nationalen Produktionsprogramme zu integrieren. Gemäss gutunterrichteter Kreise ist die Nahrungsmittelhilfepolitik von E. Saouma einer der Hauptgründe für die Unterstützung seiner Wiederwahl durch die Vereinigten Staaten (Druck der amerikanischen Agrarlobby auf die Regierung; siehe auch NZZ vom 13. Oktober 1980).

3. Bericht der Schweizer Delegation, 21. Tagung der FAO-Konferenz, Bern, Dezember 1981, Auszug aus der gemeinsamen Erklärung (Beilage 6). Uebersetzung des Autors.

4. UNDP, United Nations Development Program, Entwicklungsprogramm der Vereinten Nationen.

5. Es handelt sich um die Resolution der UNO-Vollversammlung vom November 1970 , genannt "Consensus", die die Rolle des UNDP als operationelles Koordinierungsorgan für die multilateralen Aktionen festlegt. Diese Resolution wurde durch die Resolution 35/81 vom Dezember 1980 bestätigt. Für die FAO muss jede Institution eine eigene Programmation haben und das Empfängerland ist für die Koordinierung verantwortlich. Von diesem Standpunkt aus ist es für die FAO schwer, das Patronat des UNPD zu akzeptieren. 